\title{
Impact of environmental factors and physical activity on disability and quality of life in CIDP
}

\author{
Pietro Emiliano Doneddu ${ }^{1}$. Elisa Bianchi ${ }^{2} \cdot$ Dario Cocito $^{3} \cdot$ Fiore Manganelli ${ }^{4} \cdot$ Raffaella Fazio $^{5}$. \\ Massimiliano Filosto $^{6} \cdot$ Ettore Beghi $^{2} \cdot$ Anna Mazzeo $^{7} \cdot$ Giuseppe Cosentino $^{8,9} \cdot$ Andrea Cortese $^{8,9} \cdot$ Stefano Jann $^{10}$. \\ Angelo Maurizio Clerici ${ }^{11}$. Giovanni Antonini ${ }^{12}$. Gabriele Siciliano ${ }^{13}$. Girolama Alessandra Marfia ${ }^{14}$. \\ Chiara Briani ${ }^{15}$. Giuseppe Lauria ${ }^{16,17} \cdot$ Tiziana Rosso $^{18} \cdot$ Guido Cavaletti $^{19} \cdot$ Marinella Carpo $^{20} \cdot$ Luana Benedetti $^{21}$. \\ Angelo Schenone ${ }^{22}$. Giuseppe Liberatore ${ }^{1} \cdot$ Erdita Peci $^{23} \cdot$ Emanuele Spina $^{4} \cdot$ Stefano Tronci ${ }^{5}$. \\ Stefano Cotti Piccinelli ${ }^{6}$. Antonio Toscano ${ }^{7}$. Luca Gentile ${ }^{7}$. Laura Piccolo ${ }^{8,9} \cdot$ Luca Leonardi $^{12}$. Giorgia Mataluni ${ }^{14}$. \\ Marta Ruiz ${ }^{15}$. Mario Sabatelli ${ }^{24,25}$. Lucio Santoro ${ }^{4}$ Eduardo Nobile-Orazio ${ }^{1,26}$ on behalf of the Italian CIDP \\ Database Study Group
}

Received: 8 March 2020 / Revised: 11 May 2020 / Accepted: 13 May 2020 / Published online: 19 May 2020 (c) Springer-Verlag GmbH Germany, part of Springer Nature 2020

\begin{abstract}
A few observational studies and randomized trials suggest that exercise and rehabilitation may improve activity limitation and quality of life (QoL) in patients with chronic inflammatory demyelinating polyradiculoneuropathy (CIDP), but the impact of other modifiable factors on the severity of the disease is not well understood. Using a structured questionnaire, we collected data on lifestyle and dietary habits of the patients included in the Italian CIDP database to investigate the possible influence of modifiable lifestyle factors on disability and QoL. Questionnaire data were available for 323 patients. The effect of lifestyle and dietary exposures on impairment, disability and QoL was evaluated using logistic regression models, adjusting for age, sex, disease duration, physical activity and smoking. Physical activity was associated with lower sensory impairment by the ISS scale, less disability by the INCAT and RODS scale and a better QoL in all the domains of EURO-QoL scale with the exception of anxiety/depression. None of the other parameters had an impact on these scales. This study adds evidence to the possible role of physical activity in improving symptom severity, disability and QoL in patients with CIDP. None of the other environmental factors investigated appeared to have an impact on the severity and health perception of CIDP.
\end{abstract}

Keywords Chronic inflammatory demyelinating polyradiculoneuropathy $\cdot$ CIDP $\cdot$ Physical activity $\cdot$ Epidemiology Disability · Quality of life

\section{Introduction}

Chronic inflammatory demyelinating polyradiculoneuropathy (CIDP) is an immune-mediated chronic neuropathy leading to a temporary or permanent disability in about $50 \%$ of the patients [1]. Several retrospective studies on large series of patients and a few randomized controlled trials have shown the efficacy of corticosteroids, plasma exchange, and intravenous immunoglobulin (IVIg) in CIDP [1]. However, only about $80 \%$ of patients respond to these therapies [1]. A possible influence of modifiable lifestyle components on

Eduardo Nobile-Orazio

eduardo.nobile@unimi.it

Extended author information available on the last page of the article the progression of the disease has been reported in other immune-mediated diseases including multiple sclerosis and rheumatoid arthritis [2-11]. A possible role of physical activity or rehabilitation in reducing disability has been reported in patients with inflammatory neuropathies [12-21] (Table 1), while the contribution of other potentially modifiable factors has not been investigated. The identification of these modifiable environmental factors might form the basis of a secondary preventive approach to disease management. Recently, we found that consumption of rice and fish were associated with lower risk to develop CIDP [22]. We now investigated the association of some lifestyle and dietary habits with disability, symptom severity and QoL in patients with CIDP. 


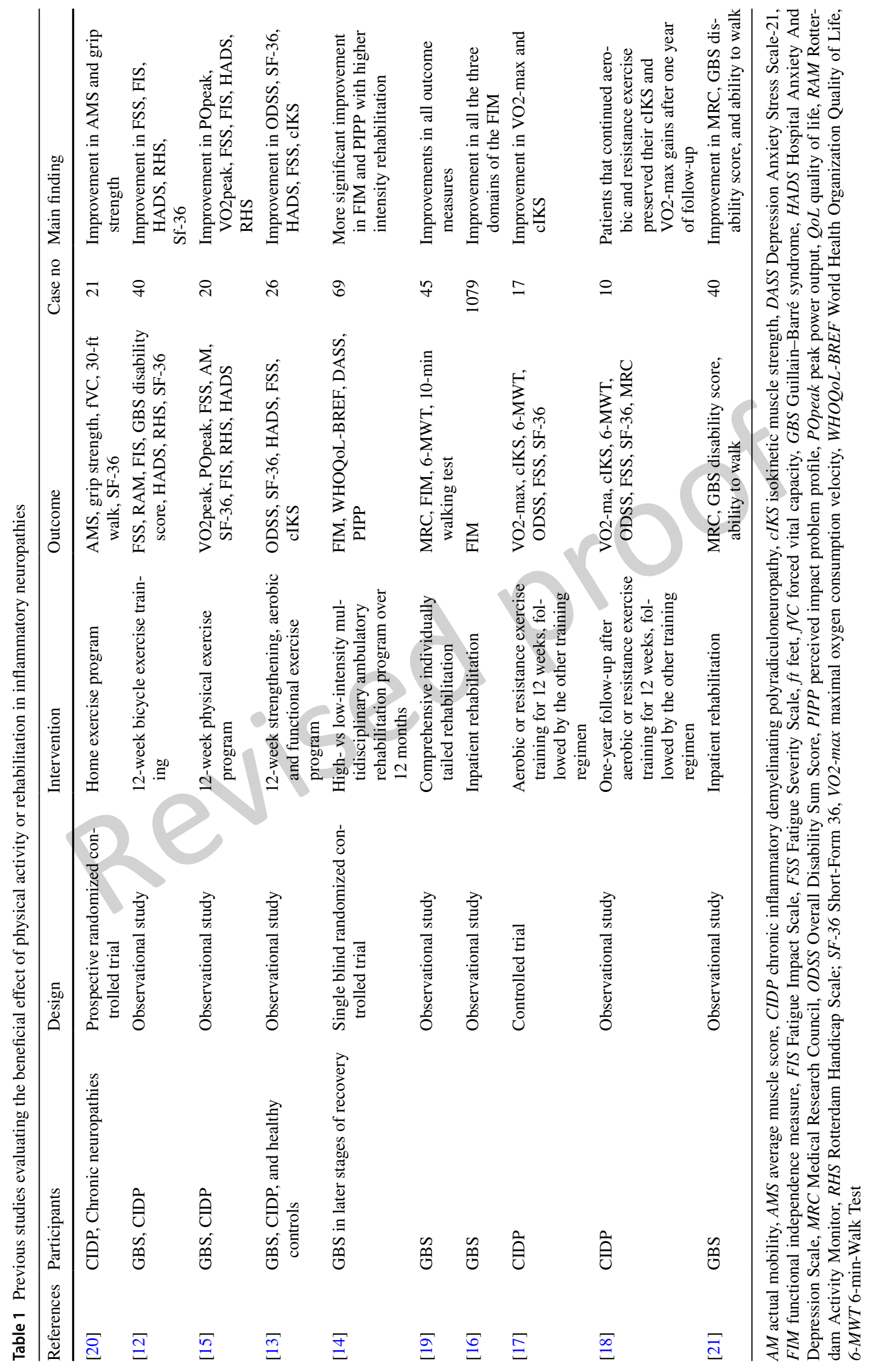




\section{Materials and methods}

\section{Study design}

We implemented a web-based database on Italian CIDP patients where, at present, data from 500 patients with a diagnosis of CIDP or one of its variants, followed by 22 Italian Centers with expertise in immune-mediated neuropathies, are included [23]. At enrollment, all eligible patients underwent a detailed clinical history including timing and distribution of neurological signs, a number of disability scales, and a QoL scale. We used the same methodology as the one employed in a previous study [23]. In this study, we collected information about lifestyle and dietary habits, related to the period after CIDP diagnosis, using a structured questionnaire. Inclusion criteria for this study were patients fulfilling the European Federation of Neurological Societies and Peripheral Nerve Society (EFNS/PNS) diagnostic criteria for CIDP [24]. Patients with a clinical diagnosis of CIDP not fulfilling the EFNS/PNS criteria were excluded, as were patients with an alternative diagnosis for the neuropathy or with increased titers of anti-MAG (myelin-associated glycoprotein) antibodies (over 7000 by Bühlman method) or without available nerve conduction studies. Only patients with an IgM monoclonal gammopathy were tested for anti-MAG antibodies. All the data were included by the treating neurologist in a web-based electronic database expressly prepared by CINECA, Bologna, Italy.

\section{Assessment of lifestyle and dietary habits}

We asked the patients for exposure to toxic agents (prolonged vs. never/occasionally), smoking (including duration and amount of exposure), illicit drug consumption (repeated vs. never/occasionally), regular alcohol use including amount of exposure (1-3 drinks per day, 4-6 drinks per day, 7-9 drinks per day, $\geq 10$ drinks per day on average), regular physical activity (type [walking for at least $30 \mathrm{~min}$, running, swimming, cycling, gymnastic, team sport, others to be specified] and frequency $[<1$ time per week, 1 time per week, 2-3 times per week, $>3$ times per week]), dietary regimen (vegan, vegetarian, macrobiotic, omnivorous, others to be specified), and frequency of consumption of a variety of foods ( 1 or more time per day, 3-4 times per week, 1-2 times per week, 2-3 times per month). Items related to dietary habits included pasta, rice, meat, raw meat, white meat, fish, vegetables, fruit, cheese, eggs, sweets, coffee, tea, milk, and soft drinks.

\section{Assessment of impairment, disability, and quality of life}

The clinical evaluation at enrollment included the following outcome measures: muscle strength measured by the
Medical Research Council (MRC) sum score on 12 muscles (rangingfrom 0 -worst to 60-best); overall sensory function measured by the INCAT sensory scale (ISS) (range from 0 - best to 20-worst); neurological disability evaluated at enrollment with the Inflammatory-Rash Overall Built Disability Scale (I-RODS) (range from 0 -worst to 48 -best) and the Inflammatory Neuropathy Cause and Treatment (INCAT) disability scale (range from $0-$ - best to 10-worst); QoL was measured using the EUROQol5D-3L scale. The scale consists of five domains, each with a score from 1-best to 3-worst that measure different aspects of QoL: mobility, self-care, usual activities, pain, anxiety/depression); the EQ VAS (Euro-QoL visual analog scale) that records the patient's self-rated health on a vertical visual analog scale, where a score of 100 refers to the best imaginable health state and a score of 0 refers to the worst imaginable health state. The VAS can be used as a quantitative measure of health outcome that reflects the patient's own judgment.

\section{Statistical analysis}

Descriptive statistics were performed on the available exposure and outcome variables in the entire sample. Data were reported as frequencies and percentages for categorical variables and as medians and interquartile ranges for continuous variables. Since most of the patients performing physical activity in our cohort reported to walk while only a minority of them performed other sport activities, physical activity was analyzed considering separately walking and performing other sport activities. The effect of lifestyle and dietary exposures on impairment, disability and QoL was evaluated using logistic regression models. The four outcomes measuring impairment (MRC, ISS) and disability (INCAT, I-RODS) were analyzed separately. For each of the four outcome measures, patients were divided into two groups based on the median of the distribution of the outcome score: (1) from 0 to the median; (2) above the median. The association was then evaluated using logistic regression models. In each model, the outcome, categorized as described above, was the dependent variable, and exposures were the independent variables. The probability modeled was that of having a higher level of impairment (MRC, ISS) or disability (INCAT, I-RODS). All models were adjusted for age, sex, disease duration (years), physical activity (yes vs. no) and smoke (yes vs. no). Models with the two disability outcomes (INCAT, I-RODS) as dependent variables were also adjusted for impairment (MRC, ISS). The association of each exposure variable with each different domain of QoL (mobility, self-care, usual activities, pain, anxiety/depression) was evaluated using separate ordinal logistic regression models, with the QoL domain (categorized as 1,2 or 3) as dependent variable, and exposures as independent variables. For the EQ VAS score, patients were divided into quartiles, 
and ordinal logistic regression models, with EQ VAS quartiles as dependent variable and exposures as independent variables, were used to evaluate the impact of each exposure variable on the EQ VAS score. The probability modeled was that of having a good level of QoL. All models were adjusted for age, sex, disease duration (years), physical activity (yes vs. no) and smoking (yes vs. no). Results obtained from each logistic regression model were expressed as odds ratio (OR) with 95\% confidence interval (CI). All tests were two-tailed and the significance level was set at 0.05 . Statistical analyses were performed with the SAS statistical package, version 9.4 (SAS Institute, Cary, NC, USA).

\section{Results}

A total of 323 patients fulfilling the EFNS/PNS criteria for CIDP were included in the analysis. Of these patients, 214 (66\%) were males and 109 (34\%) were females, with an average age at study entry of 57 years (median 58 years; range $11-89$ years) and mean disease duration of 8.5 years (median 6 years; range $0.5-52$ years). The description of exposures and outcome variables in the study sample is reported in Table 2. Logistic regression models showed that patients performing physical activity had lower impairment measured by ISS and lower disability measured by INCAT and RODS, while no association was found with MRC (Table 3). Data for QoL were available for 315 patients. Performing any physical activity was associated with higher quality of life measured by the EQ VAS and in all the EURO-QoL domains, except for anxiety/depression (Table 4). None of the remaining exposure variables revealed significant associations. The predominant type of physical activity reported by the sample was walking. Among the 155 patients performing at least one type of physical activity, a total of 64 patients only walked, while 91 were involved in at least one sport activity. A stronger association with a lower disability measured by INCAT was observed for sport activities compared to walking alone, while a similar effect of the two types of physical activity was observed on I-RODS (Table 3). When considering the EQ VAS score and mobility, self-care and pain domains of QoL, the effect of physical activity was mainly driven by sport activities, while the effect of sports on usual activities domain was similar to walking (Table 4). A positive effect on anxiety/depression domain was observed for walking, but not for other sport activities (Table 4).

\section{Discussion}

In this study, physical activity was associated with lower sensory impairment, less disability and a better QoL. Although reverse causality may have contributed to the
Table 2 Descriptive statistics of exposure and outcome variables

\begin{tabular}{|c|c|c|}
\hline Exposures & $n$ & $\%$ \\
\hline \multicolumn{3}{|l|}{ Exposure to toxic agents } \\
\hline No & 261 & 81.1 \\
\hline Yes & 61 & 18.9 \\
\hline NA & 1 & \\
\hline \multicolumn{3}{|l|}{ Physical activity } \\
\hline No & 168 & 52.0 \\
\hline Yes & 155 & 48.0 \\
\hline \multicolumn{3}{|l|}{ Type of physical activity } \\
\hline Walking & 103 & 31.9 \\
\hline Running & 10 & 3.1 \\
\hline Swimming & 29 & 9.0 \\
\hline Cycling & 34 & 10.5 \\
\hline Gym & 34 & 10.5 \\
\hline Team sport & 5 & 1.6 \\
\hline Other sport & 12 & 3.7 \\
\hline \multicolumn{3}{|l|}{ Smoke } \\
\hline No & 166 & 51.4 \\
\hline Yes & 157 & 48.6 \\
\hline \multicolumn{3}{|l|}{ Regular alcohol consumption } \\
\hline No & 204 & 63.2 \\
\hline Yes & 119 & 36.8 \\
\hline \multicolumn{3}{|l|}{ Illegal substances consumption } \\
\hline No & 311 & 98.7 \\
\hline Yes & 4 & 1.3 \\
\hline NA & 8 & \\
\hline \multicolumn{3}{|l|}{ Dietary restrictions } \\
\hline No & 298 & 92.3 \\
\hline Yes & 25 & 7.7 \\
\hline \multicolumn{3}{|l|}{ Dietary regimen } \\
\hline Omnivorous & 320 & 99.1 \\
\hline Vegetarian & 3 & 0.9 \\
\hline Impairment & Median & IQR \\
\hline MRC score & 56 & $52-60$ \\
\hline ISS score & 4 & $2-8$ \\
\hline Disability & Median & IQR \\
\hline INCAT score & 2 & $1-4$ \\
\hline I-RODS score & 35 & $26-42$ \\
\hline EURO-QOL-5D-3L $(n=315)$ & $n$ & $\%$ \\
\hline \multicolumn{3}{|l|}{ Mobility } \\
\hline 1 & 86 & 27.3 \\
\hline 2 & 221 & 70.2 \\
\hline 3 & 8 & 2.5 \\
\hline \multicolumn{3}{|l|}{ Self-care } \\
\hline 1 & 194 & 61.6 \\
\hline 2 & 111 & 35.2 \\
\hline 3 & 10 & 3.2 \\
\hline \multicolumn{3}{|l|}{ Usual activities } \\
\hline 1 & 124 & 39.4 \\
\hline 2 & 181 & 56.0 \\
\hline 3 & 10 & 3.1 \\
\hline
\end{tabular}


Table 2 (continued)

\begin{tabular}{lll}
\hline Exposures & $n$ & $\%$ \\
\hline Pain/discomfort & & \\
1 & 137 & 43.5 \\
2 & 168 & 53.3 \\
3 & 10 & 3.2 \\
Anxiety/depression & & \\
1 & 156 & 49.6 \\
2 & 145 & 46.0 \\
3 & 14 & 4.4 \\
EQ VAS (quartiles) & & \\
1 (0-49) & 51 & 17.4 \\
2 (50-59) & 62 & 21.1 \\
3 (60-74) & 106 & 36.0 \\
$4(75-100)$ & 75 & 25.5 \\
NA & 21 & \\
\hline
\end{tabular}

INCAT Inflammatory Neuropathy Cause and Treatment disability scale, IQR interquartile range, I-RODS Inflammatory-Rash Overall Built Disability Scale, ISS INCAT sensory scale, MRC Medical Research Council, EQ VAS Euro-QoL visual analogue scale, $N A$ not available

Table 3 Association of physical activity with impairment and disability

\begin{tabular}{lccc}
\hline \multicolumn{1}{c}{ OR } & $95 \%$ CI & $p$ value \\
\hline Any physical activity & & & \\
INCAT & 0.58 & $0.35-0.99$ & 0.0476 \\
I-RODS & 0.44 & $0.26-0.75$ & 0.0023 \\
MRC & 1.28 & $0.82-2.00$ & 0.2853 \\
ISS & 0.62 & $0.40-0.98$ & 0.0421 \\
Only walking & & & \\
INCAT & 0.74 & $0.37-1.47$ & 0.3933 \\
I-RODS & 0.47 & $0.23-0.78$ & 0.0335 \\
MRC & 1.63 & $0.90-2.96$ & 0.1098 \\
ISS & 0.58 & $0.31-1.05$ & 0.0727 \\
At least one sport activity & & \\
INCAT & 0.49 & $0.26-0.92$ & 0.0267 \\
I-RODS & 0.41 & $0.22-0.78$ & 0.0060 \\
MRC & 1.07 & $0.64-1.82$ & 0.7852 \\
ISS & 0.66 & $0.39-1.13$ & 0.1307 \\
\hline
\end{tabular}

CI confidence interval, INCAT Inflammatory Neuropathy Cause and Treatment disability scale, I-RODS Inflammatory-Rash Overall Built Disability Scale, ISS INCAT sensory scale, MRC Medical Research Council, $O R$ odds ratio

magnitude of the effect for disability, the association remained statistically significant after adjusting the model for the extent of impairment, suggesting that, at the same impairment level, patients who practice physical activity have less disability and better QoL.
Table 4 Association of physical activity with quality of life

\begin{tabular}{llll}
\hline & OR & $95 \%$ CI & $p$ value \\
\hline Any physical activity & & & \\
Mobility & 2.01 & $1.20-3.35$ & 0.0073 \\
Self-care & 2.05 & $1.28-3.29$ & 0.0030 \\
Usual activities & 2.17 & $1.37-3.45$ & 0.0010 \\
Pain & 1.91 & $1.21-3.02$ & 0.0053 \\
Anxiety/depression & 1.41 & $0.90-2.20$ & 0.1316 \\
EQ VAS (quartiles) & 2.08 & $1.36-3.19$ & 0.0008 \\
Only walking & & & \\
Mobility & 1.94 & $0.95-3.96$ & 0.0673 \\
Self-care & 1.79 & $0.94-3.42$ & 0.0787 \\
Usual activities & 2.62 & $1.39-4.95$ & 0.0030 \\
Pain & 1.45 & $0.80-2.63$ & 0.2260 \\
Anxiety/depression & 1.94 & $1.07-3.53$ & 0.0298 \\
EQ VAS (quartiles) & 1.97 & $1.12-3.47$ & 0.0183 \\
At least one sport activity & & & \\
Mobility & 2.57 & $1.37-4.81$ & 0.0033 \\
Self-care & 3.23 & $1.69-6.20$ & 0.0004 \\
Usual activities & 2.42 & $1.37-4.29$ & 0.0025 \\
Pain & 2.45 & $1.41-4.26$ & 0.0014 \\
Anxiety/depression & 1.14 & $0.67-1.93$ & 0.6239 \\
EQ VAS (quartiles) & 2.31 & $1.39-3.83$ & 0.0012 \\
\hline
\end{tabular}

$C I$ confidence interval, $O R$ odds ratio, EQ VAS Euro-QoL visual analog scale

Ameliorative effect of physical activity on sensory symptoms has been observed in chemotherapy-induced [25, 26] and diabetic neuropathy [27-29], and a recent study showed that physical activity was associated with less sensory neuropathy symptoms and better sensory nerve action potential amplitudes in older men [30]. Potential mechanisms include the anti-inflammatory [31] and the regenerative effect on nerve fibers of physical exercise [26, 28, 29, 32]. Previous studies have not evaluated the impact of physical activity and rehabilitation on the sensory symptoms of patients with inflammatory neuropathies (Table 1).

Our findings are also in line with the results of three previous observational studies showing that supervised aerobic cycling $[12,15]$, or unsupervised physiotherapist-prescribed community-based aerobic and strengthening exercise [13], is associated with an improvement in disability, fatigue and QoL in people with inflammatory neuropathies. A randomized controlled trial evaluating home-based exercise in patients with different chronic neuropathies including CIDP found that muscle and grip strength improved significantly [20]. These findings were subsequently confirmed by a controlled trial and its 1-year follow-up study that showed that aerobic or resistance exercise training improved strength and aerobic capacity of CIDP patients $[17,18]$, even if disability, fatigue, and QoL did not improve $[17,18]$. Since all 
participants in this study were treated at home with subcutaneous immunoglobulin, it is possible that this has negatively affected QoL and fatigue [17]. Other explanations are that participants were more severely affected than in the previous interventional studies $[12,13]$ and training programs were not focused on the weakest muscle groups in each patient. In our study, physical activity was not supervised by health personnel in the context of a study, therefore it is possible that each patient chose the type of physical activity most appropriate for his/her disturbances and that this led to a greater impact on disability and QoL.

The mechanisms underlying the improvement of muscle strength following physical activity are unknown, but could be due to an anti-inflammatory effect of exercise on the nerve lesions or muscle fiber hypertrophy or increased neural drive [17]. Future studies should investigate these underlying mechanisms in more detail using electroneuronography as well as nerve and muscle imaging.

Several studies have shown an association of diet, cigarette, alcohol consumption, and toxic agent exposure with the progression of disability and QoL in different autoimmune disorders [2-11]. We did not find an association between any of these environmental factors and the impairment, disability and QoL of patients with CIDP. One possible explanation is that the questionnaire used in our study was not able to capture the quality of diet by assessing intake of each food instead of estimating the overall dietary quality by using indicators of compliance with dietary recommendations. Future studies should evaluate the impact of diet in the progression of CIDP by using a specific questionnaire designed to capture healthy eating such as adherence to Mediterranean-style diet. We also did not find an association between rice and fish consumption and impairment and disability, suggesting that these dietary factors may influence the risk of CIDP, but not its severity [22].

The limitations of our study include the use of a non-validated lifestyle and dietary habit questionnaire. In addition, this was an observational cross-sectional retrospective study that cannot prove causality, but supports previous interventional studies showing that regular exercise may have an impact on disability and QoL in CIDP. It is also possible that CIDP patients that are severely disabled and impaired might not be able to perform physical activities and sport. Last, as this is not a population-based study, our findings might be affected by selection bias. Further epidemiological and intervention studies are needed to confirm our results in order to support a possible role of physical activity as part of a secondary preventive strategy.

Acknowledgements Italian CIDP Database study group: Pietro Emiliano Doneddu, Giuseppe Liberatore, Francesca Gallia, and Eduardo Nobile-Orazio from the Department of Medical Biotechnology and Translational Medicine, Neuromuscular and Neuroimmunology Service, Humanitas Clinical and Research Institute, Milan
University, Rozzano, Milan, Italy; Erdita Peci and Dario Cocito from the Department of Neuroscience, University of Turin, Turin, Italy. Daniele Velardo, Stefano Tronci and Raffaella Fazio from the Division of Neuroscience, Department of Neurology, Institute of Experimental Neurology (INSPE), San Raffaele Scientific Institute, Milan, Italy; Fiore Manganelli, Emanuele Spina, Antonietta Topa and Lucio Santoro from the Department of Neuroscience, Reproductive Sciences and Odontostomatology, University of Naples 'Federico II', Naples, Italy; Marta Ruiz and Chiara Briani from the Neurology Unit, Department of Neuroscience, University of Padua, Padua, Italy. Stefano Cotti Piccinelli, Alice Todeschini and Massimiliano Filosto from the Center for Neuromuscular Diseases and Neuropathies, Unit of Neurology ASST 'Spedali Civili', University of Brescia, Brescia, Italy; Corrado Cabona, Angela Zuppa and Luana Benedetti from the IRCCS Ospedale Policlinico San Martino, Genova, Italy; Antonio Toscano, Luca Gentile and Anna Mazzeo from the Department of Clinical and Experimental Medicine, Unit of Neurology, University of Messina, Messina, Italy; Giorgia Mataluni and Girolama Alessandra Marfia from the Disimmune Neuropathies Unit, Department of Systems Medicine, Tor Vergata University of Rome, Rome, Italy; Giuseppe Cosentino, Laura Piccolo, Ilaria Callegari and Andrea Cortese from the University of Pavia, IRCCS Foundation C. Mondino, Pavia, Italy; Verrengia Elena Pinuccia and Stefano Jann from the Department of Neuroscience, Niguarda Ca' Granda Hospital, Milan, Italy; Elisa Bianchi and Ettore Beghi from the Laboratorio di Malattie Neurologiche, IRCCSIstituto Mario Negri, Milan, Italy; Angelo Maurizio Clerici from the Neurology Unit, Circolo and Macchi Foundation Hospital, Insubria University, DBSV, Varese, Italy; Federica Scrascia and Marinella Carpo from the ASST Bergamo Ovest-Ospedale Treviglio, Treviglio, Italy; Martina Garnero and Angelo Schenone from the Department of Neuroscience, Rehabilitation, Ophthalmology, Genetics, Maternal and Child Health, University of Genoa and IRCCS AOU San Martino-IST, Genoa, Italy; Marco Luigetti from Fondazione Policlinico Universitario Agostino Gemelli IRCCS, UOC Neurologia, Universita' Cattolica del Sacro Cuore, Roma, Italy; Mario Sabatelli from Centro Clinico NEMO Adulti, Universita' Cattolica del Sacro Cuore, Roma, Italy; Patrizia Dacci and Giuseppe Lauria from the Unit of Neuroalgology, IRCCS Foundation "Carlo Besta" Neurological Institute, Milan, Italy; Luca Leonardi and Giovanni Antonini from the Unit of Neuromuscular Diseases, Department of Neurology Mental Health and Sensory Organs (NESMOS), Faculty of Medicine and Psychology, 'Sapienza' University of Rome, Sant'Andrea Hospital, Rome, Italy; Tiziana Rosso from the Azienda UL.SS. 8 Asolo, Castelfranco Veneto, Italy; Erika Schirinzi and Gabriele Siciliano from the Neurology Unit, Department of Clinical and Experimental Medicine, University of Pisa, Pisa, Italy; Claudia Balducci and Guido Cavaletti from the School of Medicine and Surgery and Experimental Neurology Unit, University of MilanoBicocca, Monza, Italy.

Author contributions PED designed and conceptualized the study, had a major role in the acquisition of data, analyzed the data, and wrote the first draft of the manuscript. EBianchi designed and executed the statistical analysis, contributed to the conception, organization, and execution of the research project, and reviewed and commented on the statistical analysis and the report. DC, FM, RF, MF, EBeghi, AM, GC, AC, SJ, AMC, GA, GS, GAM, CB, GL, TR, GC, MC, LB, AS, GL, EP, ES, ST, SCP, AT, LG, LP, EPV, LL, ES, GM, MR, MS and LS contributed to the study conception and design, had a major role inacquisition and interpretation of data and revised the manuscript for intellectual content. ENO conceived, organized and designed the study, reviewed and commented on the statistical analysis and reviewed the report.

Funding The study was supported by a grant from Regione Lombardia, Italy, for patients from this region and subsequently extended to other Italian centers. The study was also supported by unrestricted grants 
from Kedrion Biopharma (Italy), CSL Behring (Italy), Humanitas Clinical and Research Institute (Milan, Italy), and GBS-CIDP Foundation International (USA). The funders had no role in study design, data collection and analysis, decision to publish, or in the preparation of the manuscript.

Data availability The data that support the findings of this study are available from the corresponding author, upon request.

\section{Compliance with ethical standards}

Conflicts of interest Pietro Emiliano Doneddu has received travel grants to attend scientific meetings from CSL Behring and Kedrion. Dario Cocito has received honoraria for lecturing fromShire, CSL Behring, and Kedrion and travel grants to attend scientific meeting from Shire, Kedrion, and CSL Behring. Fiore Manganelli reports personal fees for scientific events from CSL Behring and has received travel grants to attend scientific meetings from CSL Behring and Kedrion. Raffaella Fazio has served on scientific advisory boards for CSL Behring and has received travel grants from Kedrion and CSL Behring to attend scientific meeting. Chiara Briani has served on scientific advisory boards for Pfizer, Alnylam, and Akcea, and has received travel grants from Kedrion and CSL Behring to attend scientific meeting. Massimiliano Filosto has served on scientific advisory boards for CSL Behring and has received travel grants from Kedrion, Baxter and CSL Behring to attend scientific meeting. Stefano Jann has received research grants from Grifols, outside this work, and travel grants from Grifols and Kedrion. Anna Mazzeo has received travel grants from Kedrion and CSL Behring to attend scientific meeting. Giuseppe Cosentino has received travel grants to attend scientific meetings from CSL Behring and Kedrion. Andrea Cortese has received travel grants to attend scientific meetings from Kedrion. Marinella Carpo has received travel grants to attend scientific meetings from Kedrion. Guido Cavaletti has received honoraria for lecturing and travel grants to attend scientific meetings from Kedrion. Ettore Beghi reports grants from UCB-Pharma, grants from Shire, grants from EISAI, personal fees from Viropharma, grants from Italian Ministry of Health, grants from Fondazione Borgonovo, grants from Associazione IDIC 15 and grants from European Union, outside the submitted work. Giuseppe Liberatore has received travel grants to attend scientific meetings from CSL Behring and Kedrion. Lucio Santoro reports personal fees for scientific events from CSL Behring and has received travel grants to attend scientific meetings from CSL Behring and Kedrion. Erdita Peci has received travel grants to attend scientific meetings from CSL Behring. Eduardo Nobile Orazio reports personal fees for Advisory or Scientific Board from Kedrion, Italy, Baxter, Italy, Novartis, Switzerland, CSL-Behring, Italy, Astellas, the Netherlands, outside the submitted work and travel grants to attend Scientific Meeting from Baxter, Grifols, Kedrion, and Novartis, Italy. The other authors declare no conflict of interest.

Ethics approval The study was approved by the Ethical Committee of each participating center.

Consent to participate Written informed consent was obtained from all participants at enrollment.

\section{References}

1. Nobile-Orazio E (2014) Chronic Inflammatory demyelinating polyradiculoneuropathy. Where we are, where we should go. J Peripher Nerv Syst 19:2-13. https://doi.org/10.1111/jns5.12053
2. Jelinek GA, De Livera AM, Marck CH, Brown CR, Neate SL, Taylor KL et al (2016) Associations of lifestyle, medication, and socio-demographic factors with disability in people with multiple sclerosis: an international cross-sectional study. PLoS ONE 25(11):e0161701. https://doi.org/10.1371/journal.pone.0161701

3. Belbasis L, Bellou V, Evangelou E, Ioannidis JPA, Tzoulaki I (2015) Environmental risk factors and multiple sclerosis: an umbrella review of systematic reviews and meta-analyses. Lancet Neurol 14:263-273. https://doi.org/10.1016/S1474 $-4422(14) 70267-4$

4. Manzel A, Muller DN, Hafler DA, Erdman SE, Linker RA, Kleinewietfeld M (2014) Role of "Western Diet" in inflammatory autoimmune diseases. Curr Allergy Asthma Rep 14:404. https://doi.org/10.1007/s11882-013-0404-6

5. Fitzgerald KC, Tyry T, Salter A, Cofield SS, Cutter G, Fox R et al (2018) Diet quality is associated with disability and symptom severity in multiple sclerosis. Neurology 90:1-11. https:// doi.org/10.1212/WNL.0000000000004768

6. Harel-Meir M, Sherer Y, Shoenfeld Y (2007) Tobacco smoking and autoimmune rheumatic diseases. Nat Clin Pract Rheumatol 3:707-715. https://doi.org/10.1038/ncprheum0655

7. Weiland TJ, Hadgkiss EJ, Jelinek GA, Pereira NG, Marck CH, van der Meer DM (2014) The association of alcohol consumption and smoking with quality of life, disability and disease activity in an international sample of people with multiple sclerosis. J Neurol Sci 336:211-219. https://doi.org/10.1016/j. jns.2013.10.046

8. Bombardier CH, Blake KD, Ehde DM, Gibbons LE, Moore D, Kraft GH (2004) Alcohol and drug abuse among persons with multiple sclerosis. Mult Scler 10:35-40. https://doi. org/10.1191/1352458504ms989oa

9. Beier M, D'Orio V, Spat J, Shuman M, Foley FW (2014) Alcohol and substance use in multiple sclerosis. J Neurol Sci 338:122-127. https://doi.org/10.1016/j.jns.2013.12.029

10. Swanson GR, Sedghi S, Farhadi A, Keshavarzian A (2010) Pattern of alcohol consumption and its effect on gastrointestinal symptoms in inflammatory bowel disease. Alcohol 44:223-228. https ://doi.org/10.1016/j.alcohol.2009.10.019

11. Stejskal J, Stejskal VD (1999) The role of metals in autoimmunity and the link to neuroendocrinology. Neuro Endocrinol Lett 20:351-364

12. Garssen MP, Bussmann JB, Schmitz PI, Zandbergen A, Welter TG, Merkies IS et al (2004) Physical training and fatigue, fitness, and quality of life in Guillain-Barré syndrome and CIDP. Neurology 63:2393-2395. https://doi.org/10.1212/01.WNL.0000148589 $.87107 .9 \mathrm{C}$

13. Graham RC, Hughes RA, White CM (2007) A prospective study of physiotherapist prescribed community based exercise in inflammatory peripheral neuropathy. J Neurol 254:228-235. https://doi. org/10.1007/s00415-006-0335-4

14. Khan F, Pallant JF, Amatya B, Ng L, Gorelik A, Brand C (2011) Outcomes of high- and low-intensity rehabilitation programme for persons in chronic phase after Guillain-Barré syndrome: a randomized controlled trial. J Rehabil Med 43:638-646

15. Bussmann JB, Garssen MP, van Doorn PA, Stam HJ (2007) Analysing the favourable effects of physical exercise: relationships between physical fitness, fatigue and functioning in Guillain-Barré syndrome and chronic inflammatory demyelinating polyneuropathy. J Rehabil Med 39:121-125

16. Andrews AW, Middleton A (2018) Improvement during inpatient rehabilitation among older adults with Guillain-Barré syndrome, multiple sclerosis, Parkinson disease, and stroke. Am J Phys Med Rehabil 97:879-884

17. Markvardsen LH, Overgaard K, Heje K, Sindrup SH, Christiansen I, Vissing J et al (2018) Resistance training and aerobic training improve muscle strength and aerobic capacity in chronic 
inflammatory demyelinating polyneuropathy. Muscle Nerve 57:70-76. https://doi.org/10.1002/mus.25652

18. Markvardsen LK, Carstens AR, Knak KL, Overgaard K, Vissing J, Andersen H (2019) Muscle strength and aerobic capacity in patients with CIDP one year after participation in an exercise trial. J Neuromuscul Dis 6:93-97

19. Novak P, Šmid S, Vidmar G (2017) Rehabilitation of GuillainBarré syndrome patients: an observational study. Int J Rehabil Res 40:158-163

20. Ruhland JL, Shields RK (1997) The effects of a home exercise program on impairment and health-related quality of life in persons with chronic peripheral neuropathies. Phys Ther 77:1026-1039

21. Prada V, Massa F, Salerno A, Fregosi D, Beronio A, Serrati C et al (2020) Importance of intensive and prolonged rehabilitative treatment on the Guillain-Barrè syndrome long-term outcome: a retrospective study. Neurol Sci 41:321-327

22. Doneddu PE, Bianchi E, Cocito D, Manganelli F, Fazio R, Filosto $M$ et al (2020) Risk factors for chronic inflammatory demyelinating polyradiculoneuropathy (CIDP): antecedent events, lifestyle and dietary habits. Data from the Italian CIDP Database. Eur J Neurol 27:136-143. https://doi.org/10.1111/ene.14044

23. Doneddu PE, Cocito D, Manganelli F, Fazio R, Briani C, Filosto $M$ et al (2019) Atypical CIDP: diagnostic criteria, progression and treatment response. Data from the Italian CIDP Database. J Neurol Neurosurg Psychiatry 90:125-132. https://doi.org/10.1136/ jnnp-2018-318714

24. Joint Task Force of the EFNS and the PNS (2010) European Federation of Neurological Societies/Peripheral Nerve Society Guideline on management of chronic inflammatory demyelinating polyradiculoneuropathy: report of a joint task force of the European Federation of Neurological Societies and the Peripheral Nerve Society First Revision. J Peripher Nerv Syst 15:1-9. https ://doi.org/10.1111/j.1529-8027.2010.00245.x

25. Zimmer P, Trebing S, Timmers-Trebing U, Schenk A, Paust R, Bloch W et al (2018) Eight-week, multimodal exercise counteracts a progress of chemotherapy-induced peripheral neuropathy and improves balance and strength in metastasized colorectal cancer patients: a randomized controlled trial. Support Care Cancer 26:615-624. https://doi.org/10.1007/s00520-017-3875-5

26. Streckmann F, Lehmann HC, Balke M, Schenk A, Oberste M, Heller A et al (2018) Sensorimotor training and whole-body vibration training have the potential to reduce motor and sensory symptoms of chemotherapy-induced peripheral neuropathy-a randomized controlled pilot trial. Support Care Cancer 27:2471-2478. https ://doi.org/10.1007/s00520-018-4531-4

27. Gu Y, Dennis SM, Kiernan MC, Harmer AR (2018) Aerobic exercise training may improve nerve function in type 2 diabetes and pre-diabetes: a systematic review. Diabetes Metab Res Rev 35:e3099. https://doi.org/10.1002/dmrr.3099

28. Cooper MA, Kluding PM, Wright DE (2016) Emerging relationships between exercise, sensory nerves, and neuropathic pain. Front Neurosci 10:372. https://doi.org/10.3389/fnins.2016.00372

29. Kluding PM, Pasnoor M, Singh R, Jernigan S, Farmer K, Rucker $\mathrm{J}$ et al (2012) The effect of exercise on neuropathic symptoms, nerve function, and cutaneous innervation in people with diabetic peripheral neuropathy. J Diabet Complicat 26:424-429. https:// doi.org/10.1016/j.jdiacomp.2012.05.007

30. Lange-Maia BS, Cauley JA, Newman AB, Boudreau RM, Jakicic JM, Glynn NW et al (2016) Sensorimotor peripheral nerve function and physical activity in older men. J Aging Phys Act 24:559566. https://doi.org/10.1123/japa.2015-0207

31. Sharif K, Watad A, Bragazzi NL, Lichtbroun M, Amital H, Shoenfeld Y (2018) Physical activity and autoimmune diseases: get moving and manage the disease. Autoimmun Rev 17:53-72. https ://doi.org/10.1016/j.autrev.2017.11.010

32. Feter N, Freitas MP, Gonzales NG, Umpierre D, Cardoso RK, Rombaldi AJ (2018) Effects of physical exercise on myelin sheath regeneration: a systematic review and meta-analysis. Sci Sports 33:8-21. https://doi.org/10.1016/j.scispo.2017.06.009

\section{Affiliations}

Pietro Emiliano Doneddu ${ }^{1}$ - Elisa Bianchi ${ }^{2} \cdot$ Dario Cocito $^{3} \cdot$ Fiore Manganelli $^{4} \cdot$ Raffaella Fazio $^{5}$. Massimiliano Filosto ${ }^{6}$. Ettore Beghi ${ }^{2} \cdot$ Anna Mazzeo $^{7} \cdot$ Giuseppe Cosentino $^{8,9} \cdot$ Andrea Cortese $^{8,9} \cdot$ Stefano Jann $^{10}$. Angelo Maurizio Clerici ${ }^{11}$. Giovanni Antonini ${ }^{12}$. Gabriele Siciliano ${ }^{13}$. Girolama Alessandra Marfia ${ }^{14}$. Chiara Briani ${ }^{15}$. Giuseppe Lauria ${ }^{16,17} \cdot$ Tiziana Rosso $^{18}$. Guido Cavaletti ${ }^{19}$ - Marinella Carpo ${ }^{20} \cdot$ Luana Benedetti $^{21}$. Angelo Schenone ${ }^{22}$. Giuseppe Liberatore ${ }^{1} \cdot$ Erdita Peci $^{23} \cdot$ Emanuele Spina $^{4} \cdot$ Stefano Tronci ${ }^{5}$. Stefano Cotti Piccinelli ${ }^{6}$. Antonio Toscano ${ }^{7}$. Luca Gentile ${ }^{7} \cdot$ Laura Piccolo $^{8,9}$. Luca Leonardi ${ }^{12}$. Giorgia Mataluni ${ }^{14}$. Marta Ruiz ${ }^{15}$. Mario Sabatelli ${ }^{24,25}$. Lucio Santoro ${ }^{4}$ Eduardo Nobile-Orazio ${ }^{1,26}$ on behalf of the Italian CIDP Database Study Group

1 Neuromuscular and Neuroimmunology Service, Humanitas Clinical and Research Center-IRCCS, Rozzano, Milan, Italy

2 Laboratorio di Malattie Neurologiche, Istituto di Ricerche Farmacologiche Mario Negri IRCCS, Milan, Italy

3 Istituti Clinici Scientifici Maugeri, Turin, Italy

4 Department of Neuroscience, Reproductive Sciences and Odontostomatology, University of Naples 'Federico II', Naples, Italy

5 Division of Neuroscience, Department of Neurology, Institute of Experimental Neurology (INSPE), San Raffaele Scientific Institute, Milan, Italy
6 Center for Neuromuscular Diseases and Neuropathies, Unit of Neurology, ASST 'Spedali Civili', University of Brescia, Brescia, Italy

7 Department of Clinical and Experimental Medicine, Unit of Neurology, University of Messina, Messina, Italy

8 University of Pavia, Pavia, Italy

9 IRCCS Mondino Foundation, Pavia, Italy

10 Department of Neuroscience, Niguarda Ca' Granda Hospital, Milan, Italy

11 Neurology Unit, Circolo and Macchi Foundation Hospital, DBSV, Insubria University, Varese, Italy 
12 Department of Neurology Mental Health and Sensory Organs (NESMOS), Faculty of Medicine and Psychology, Unit of Neuromuscular Diseases, 'Sapienza' University of Rome, Sant'Andrea Hospital, Rome, Italy

13 Department of Clinical and Experimental Medicine, Neurology Unit, University of Pisa, Pisa, Italy

14 Department of Systems Medicine, Dysimmune Neuropathies Unit, Tor Vergata University of Rome, Rome, Italy

15 Department of Neuroscience, Neurology Unit, University of Padua, Padua, Italy

16 Unit of Neuroalgology, IRCCS Foundation 'Carlo Besta' Neurological Institute, Milan, Italy

17 Department of Biomedical and Clinical Sciences 'Luigi Sacco', University of Milan, Milan, Italy

18 ULSS2 Marca Trevigiana, UOC Neurologia-Castelfranco Veneto, Treviso, Italy
19 School of Medicine and Surgery and Experimental Neurology Unit, University of Milano-Bicocca, Monza, Italy

20 ASST Bergamo Ovest-Ospedale Treviglio, Treviglio, Italy

21 IRCCS Ospedale Policlinico San Martino Genova, Genoa, Italy

22 Department of Neuroscience, Rehabilitation, Ophthalmology, Genetics, Maternal and Child Health, University of Genoa and IRCCS AOU San Martino-IST, Genoa, Italy

23 Department of Neuroscience, University of Turin, Turin, Italy

24 Università Cattolica del Sacro Cuore. Sede di Roma, Rome, Italy

25 Centro Clinico NEMO Adulti, Rome, Italy

26 Department of Medical Biotechnology and Translational Medicine (BIOMETRA), Milan University, Milan, Italy 